\title{
Circulaciones laborales de mujeres migrantes en Buenos Aires: de empleadas domésticas a enfermeras*
}

\author{
Ana Inés Mallimaci Barral**
}

\begin{abstract}
Resumen
Este artículo se enmarca en un trabajo de investigación más amplio sobre las circulaciones laborales de las mujeres migrantes internacionales en el sector de cuidados argentino. Específicamente, se centrará en el análisis de dos trayectorias de mujeres que en la actualidad se desempeñan como enfermeras. A partir de la realización de entrevistas en profundidad, se intenta analizar las trayectorias laborales en su relación con los proyectos migratorios, la vinculación establecida con el trabajo de cuidados (remunerado y no remunerado) y las representaciones de las propias mujeres sobre su carrera laboral.
\end{abstract}

Palabras clave: Migraciones, Cuidado, Empleo doméstico, Enfermería, Argentina.

* Recibido el 06 de diciembre de 2017, aceptado el 26 de junio de 2018.

** Investigadora Adjunta del Consejo Nacional de Investigaciones Científicas y Técnicas (CONICET) en el Instituto Interdisciplinario de Estudios de género de la Universidad de Buenos Aires (IIEGE-UBA); Profesora adjunta regular de la Universidad Nacional Arturo Jauretche, Buenos Aires, Argentina. anamallimaci@gmail.com / https://orcid.org/0000-0001-9007-895X 
Labor Circulation of Migrant Women in Buenos Aires: from Domestic Workers to Nurses

\begin{abstract}
This article is part of a broader study about the labor circulations of international migrant women in the Argentine care sector. Specifically, it focuses on the analysis of the trajectories of two women who work as nurses. In-depth interviews are used to understand the labor trajectories and their relationship with migratory projects, the ties with the work of care (paid and unpaid) and the representations of the women about their career.
\end{abstract}

Keywords: Migrations, Care, Domestic Work, Nursing, Argentina. 


\section{Introducción}

Al igual que en muchos países de América Latina, en Argentina los movimientos migratorios son parte constitutiva de la conformación de los Estados y sociedades nacionales. Pueden identificarse dos movimientos principales: uno de mayor intensidad y excepcionalidad relacionado con el arribo de contingentes europeos que lideraron en número a la población migratoria del país desde fines del Siglo XIX hasta mediados del Siglo XX, y un segundo movimiento de mayor estabilidad $\mathrm{y}$ constancia representado por la población proveniente de países sudamericanos. En la actualidad, Argentina es el país con mayor proporción de migrantes intrarregionales de Sudamérica. En consecuencia, las migraciones han sido y son un elemento central de análisis de las ciencias sociales argentinas que conformaron un campo consolidado de estudios migratorios.

Los estudios de género han producido transformaciones dentro de este campo generando importantes trabajos, especialmente sobre mujeres migrantes. Estos estudios han mostrado que históricamente las mujeres migrantes en el país se han desempeñado en un número reducido de empleos, entre los cuales se destaca el empleo doméstico. El análisis de la dimensión laboral de las migraciones femeninas ha incluido el efecto de la permanencia en sus trayectorias laborales (Maguid y Bruno 2010), las formas de movilidad laboral que representan (Cerrutti y Bruno 2006) y su participación en el trabajo doméstico como refugio laboral (Rosas et alii, 2015).

En cuanto a la relación entre enfermería y migraciones, existe un importante desarrollo de trabajos, especialmente sobre la migración de enfermeras en contextos de globalización y su impacto para los servicios de salud de los países de origen y de destino (Malvárez 2007, Malvárez y Castrillón, 2005, Rodríguez, Muñoz y Hoga, 2014, OPS 2011a). Si bien existen enfermeras que migran hacia la Argentina (Según un informe de la OPS publicado en el 2011 el número asciende a 211), se trata de un fenómeno reducido si se lo compara con las enfermeras migrantes que se han formado 
en el país (Mallimaci Barral, 2016). De esta manera, este artículo no trata de la migración de profesionales de la salud (temporales o permanentes) sino de estrategias de la población migrante residente en el país de acuerdo con las oportunidades de trabajo ofrecidas y la aspiración a cierta movilidad social ascendente.

En las páginas que siguen, se intenta analizar los recorridos laborales de las mujeres migrantes desde el punto de vista de sus protagonistas. Con el fin de poder indagar en las circulaciones laborales y en la posibilidad de generar una "carrera laboral" dentro del sector de cuidados, se seleccionaron dos casos de mujeres enfermeras que se desempeñaron como trabajadoras domésticas. Este tipo de trayectorias nos ubica en situaciones poco frecuentes para las migrantes internacionales en Argentina, tanto por la circulación entre diferentes sectores de trabajo como por el acceso a un empleo profesional a partir del estudio de una carrera en el contexto de migración. Se trata, en definitiva, del análisis de trayectorias condicionadas social y económicamente pero que eluden las formas más previsibles de sus secuencias.

\section{Metodología}

Este artículo es parte de una investigación más amplia sobre migraciones y cuidado en la Argentina ${ }^{1}$, en la que se reconstruyeron carreras laborales retrospectivas de las entrevistadas que trabajan en el sector de los cuidados remunerados para "reconstruir la vida laboral de las actoras sociales a posteriori, interesándose en las entrevistas por el trayecto anterior de la vida de los sujetos y dando preeminencia al relato de sus recorridos pasados" (Muñiz Terra, 2013:61). Se utiliza la noción de "carrera" que se refiere a "la secuencia de movimientos de un puesto de trabajo a otro que hace un individuo que se desplaza dentro del sistema ocupacional" (Becker,

1 Esta investigación se encuentra enmarcada en el Proyecto PIP "Mujeres migrantes y la gestión del cuidado en Buenos Aires y Córdoba" (PIP $11220130100528 \mathrm{CO})$, financiado por el CONICET Argentina y dirigido por Ana Inés Mallimaci Barral. 
2009 en Tizziani, 2011). Muñiz Terra (2012) define la noción de carreras laborales como parte de una perspectiva teórica que le otorga un rol central a los actores sociales al enfocarse en el encadenamiento de secuencias en la vida laboral de los actores. A través de estas transiciones se pueden ver las intenciones de los actores, sus representaciones, su desarrollo complejo, pero, sobre todo, dinámico (Muñiz Terra, 2012). Partiendo de este tipo de herramientas metodológicas es posible vincular las características de la estructura social y del mercado de trabajo local, es decir su etnificación, racialización y generización que organiza el horizonte de posibilidades laborales que se les abre a las mujeres migrantes (Tizziani, 2011) y que se encuentran necesariamente vinculadas con las trayectorias biográficas, migratorias y familiares.

Analizar movimientos laborales desde técnicas metodológicas que no partan de ciertos parámetros predefinidos para medir el efectivo ascenso / permanencia / o descenso en las calificaciones laborales, le otorga una relevancia central a la percepción que tienen de estos movimientos las propias mujeres migrantes con el fin de comprender el rol que cumplen estos empleos en sus trayectorias. En este sentido, la noción de circulación y movilidad dentro de las carreras laborales utilizada en este trabajo, no se halla definida por dimensiones objetivas como la pertenencia a una clase (y el corrimiento de la misma) o la posesión de ciertas calificaciones educativas y laborales, sino que remite a una experiencia subjetiva y procesual, un modo en que las mujeres relatan sus propias trayectorias y decisiones. De esta manera, tal como lo señala Dalle (2013), se concibe a la movilidad social como un proceso y no simplemente como la comparación entre la posición de clase de partida y de llegada. Sin embargo, esta experiencia subjetiva se sustenta necesariamente en el acceso a espacios, puestos y posibilidades que tienen reconocimientos diferenciales en términos de legitimidad, respetabilidad y el acceso a derechos.

Se realizaron 18 entrevistas con enfermeras extranjeras que se desempeñan en hospitales públicos y clínicas privadas del Gran Buenos Aires (GBA) y estudiantes de enfermería pertenecientes a 
dos universidades nacionales y dos escuelas privadas. En esos mismos contextos también fueron entrevistadas mujeres argentinas, muchas de ellas oriundas de otras provincias del país (10 migrantes internas y 10 nacidas en el GBA). Asimismo, el trabajo se complementa con entrevistas, algunas grupales, a coordinadores/as del sector de enfermería en el ámbito profesional, docentes y rectores de las instituciones educativas (11 personas entrevistadas) y dos sindicalistas. Entre las mujeres extranjeras, la totalidad llega a la Argentina en migraciones familiares entendiendo por ello desplazamientos vinculados a cadenas familiares. Las principales divergencias son las edades, dado que 13 llegan siendo niñas y acceden a la enfermería después de una socialización en instituciones educativas nacionales. Sin embargo, en las estadísticas nacionales se consideran "extranjeras" por su nacimiento en otro país. El resto son mujeres adultas que llegan a la argentina con fines laborales y se insertan en empleos vinculados a los cuidados.

Las historias que serán analizadas en este trabajo pertenecen a dos mujeres extranjeras que, a lo largo de su carrera laboral, circularon por diferentes empleos vinculados al cuidado remunerado y que lograron atravesar la barrera de la informalidad a partir de la formación en una carrera profesional (del empleo doméstico a la enfermería). La definición de un sector laboral relacionado con el cuidado supone compartir una noción amplia de "cuidado" que involucre al conjunto de actividades remuneradas que giran en torno al sostén cotidiano de la vida humana en el marco de dos dimensiones centrales: las disposiciones y motivaciones ético-afecticas y las tareas concretas de la vida diaria (Vega y Gutiérrez-Rodríguez, 2014:9-10). Este tipo de trayectorias es compartido por otras mujeres entrevistadas, extranjeras y argentinas. Con el fin de poder profundizar en los procesos singulares de las trayectorias analizadas, se seleccionaron únicamente dos casos para poder detenerse en las particularidades que suelen quedar opacadas cuando se analiza una mayor cantidad de entrevistas. Se trata de un ejercicio interpretativo que permite avanzar en la comprensión de las formas en que las 
trayectorias analizadas se estructuran alrededor de determinados ejes.

\section{Presentación de casos}

Lejos de intentar generalizar los resultados de este análisis, el principal propósito de estas páginas consiste en comprender trayectorias donde las migrantes transitan carreras laborales que se alejan de los destinos naturalizados por ellas y por la sociedad más amplia. Como se verá, a partir de una profunda conciencia y reflexividad sobre su posición en la sociedad (Borgeaud Garciandia 2014, Skegg 2015), estas mujeres van tomando decisiones pragmáticas en un horizonte acotado de posibilidades. Uno de los resultados obtenidos en la investigación más amplia muestra que para el caso del GBA existen dos formas típicas en que las mujeres extranjeras acceden al mundo de la enfermería:

\section{a. Movilidad intergeneracional}

Un primer caso lo representan mujeres que han migrado en contextos familiares cuando eran pequeñas. Se trata de mujeres nacidas en el extranjero (inmigrantes de acuerdo con las categorías del sistema estadístico nacional) pero que han crecido y estudiado en el país. A lo largo de la permanencia en Argentina y la inserción y especialización familiar en un sector productivo (relacionados con los cuidados en casas particulares, la construcción, el sector textil y/o horticultura) las familias han experimentado una movilidad ascendente, especialmente en términos económicos. La posibilidad de contar con recursos monetarios y las aspiraciones familiares de ampliar las opciones para las generaciones siguientes producen las condiciones de posibilidad que permiten el estudio terciario $y / 0$ universitario de estas mujeres. 
b. Circulaciones entre empleos de cuidado

Se trata de mujeres migrantes que se desempeñaron o desempeñan en casas particulares como empleadas encargadas de limpieza o como cuidadoras de niños/as o personas mayores que a partir de un número variado de "pasajes" laborales acceden a ser enfermeras. A diferencia del primer caso, se trata de un ascenso que se experimenta a lo largo de la propia trayectoria migratoria y laboral.

Se seleccionaron dos casos, Jimena y $V_{i v i a n a}^{2}$, que pertenecen al segundo tipo con la intención de poder analizar con detalle la forma y el sentido de las circulaciones laborales dentro del mundo de los empleos relacionados con los cuidados.

Viviana tiene 29 años, nació en un pueblo llamado San Isidro en el departamento de Itapúa, cercano a la ciudad de Posadas. Es la menor de 9 hermanos. A su mamá la define como una ama de casa que "hacía otros trabajos" como trabajos de costura y de "enfermería como las de antes", es decir, de modo no profesional o "empírico". El papá siempre trabajó en la construcción. Viviana pertenece a la larga tradición de la migración paraguaya en Argentina, la más importante en términos cuantitativos dentro del universo de migrantes regionales en todo el país desde el año 1947 (Benencia, 2012). La migración paraguaya ha tenido tradicionalmente una proporción de mujeres elevada: el Índice de masculinidad era de 101, 8 varones por cada 100 mujeres en 1960, lo que da cuenta de una paridad entre las mujeres y varones migrantes, que desciende a 85,6 por ciento en 1980, 73,5 en 1990 y aumenta a 79,7 en el 2010. Una parte importante de las mujeres paraguayas se inserta tradicionalmente en el trabajo doméstico de las grandes ciudades, especialmente en Buenos Aires.

Jimena tiene 51 años y nació en Oruro, Bolivia, en un pueblo "chico" que estaba organizado en torno a la actividad

${ }^{2}$ Los nombres han sido modificados para mantener el anonimato de las entrevistadas y la confidencialidad de sus relatos. 
minera. Vivía con sus padres y sus 12 hermanos/as, ella ocupa el sexto lugar. Su padre era ferroviario y trabajaba en el lavado de estaño. Su madre también participaba en la actividad minera, pero a partir de su maternidad se dedicó al trabajo doméstico. Jimena forma parte de la población boliviana, la segunda en importancia en el país. La presencia masculina es más importante que entre la migración paraguaya, aun cuando exista un efectivo aumento de la presencia de mujeres en la composición total de la migración: el índice de masculinidad pasa de 125,4 por ciento en 1980 a 98,7 por ciento en el 2010 (Benencia, 2012). Pero sin duda, la mayor diferencia entre las tradiciones migratorias paraguayas y bolivianas radica en cuáles han sido los proyectos migratorios femeninos más comunes: mientras que el "tipo ideal" de mujer boliviana migrante llega a nuestro país a través de relaciones familiares previas y, en el caso de estar en pareja, suele ser el varón el encargado del movimiento pionero hacia la Argentina, entre las mujeres paraguayas existe una mayor proporción de mujeres pioneras. En cuanto a las inserciones laborales, el trabajo doméstico tiene mayor incidencia entre las mujeres paraguayas que entre las bolivianas que diversifican sus opciones laborales en producciones familiares vinculadas al sector textil, el comercio minorista y la agricultura.

En este sentido, Viviana y Jimena representan en sus formas de migrar los arquetipos de la migración de mujeres correspondientes a su nacionalidad: Viviana viaja sin pareja con el proyecto, resuelto por sus familiares ya establecidos en Buenos Aires, de insertarse en el empleo doméstico remunerado. Jimena, casada y con una hija, migra para reunirse con su pareja sin que ello signifique la ausencia de un proyecto laboral autónomo (como se ha mostrado en Mallimaci Barral, 2011).

En la actualidad, ambas son enfermeras y trabajan en hospitales públicos de la Ciudad de Buenos Aires. Jimena es licenciada en enfermería, estudió enfermería profesional y luego la licenciatura en instituciones privadas. Viviana es enfermera profesional y está estudiando la licenciatura en enfermería en una 
universidad nacional de la Provincia de Buenos Aires. Las dos fueron entrevistadas en el año 2016 en la Ciudad de Buenos Aires.

En los siguientes apartados se analizarán con mayor detalle las trayectorias migratorias de las entrevistadas visibilizando las múltiples dimensiones de sus recorridos migratorios y laborales.

\section{Análisis de las trayectorias}

Los relatos que brindan las entrevistadas contienen una riqueza y exhaustividad cuyo análisis superaría ampliamente los límites de espacio exigidos en este artículo. Por ello, se seleccionaron aquellas dimensiones comunes que permiten comprender algunos aspectos de los recorridos singulares de sus trayectorias.

\section{a. Origen social y trayectoria migratoria}

Jimena es la única de su familia que emigró hacia la Argentina, lo que marca una diferencia importante con las historias comunes de las familias bolivianas migrantes, que están signadas por la migración internacional como una opción ordinaria y rutinaria dentro de las estrategias disponibles de reproducción de los hogares que se expresa en largas tradiciones familiares de migración hacia la Argentina (Mallimaci, 2012; Hinojosa, 2009). Sin embargo, recuerda que migrar hacia la Argentina era una experiencia común en su pueblo, especialmente desde la década de los 80 s con la crisis de la actividad minera y la caída internacional del precio del Estaño. Jimena finaliza la escuela secundaria en Bolivia, un rasgo excepcional en su biografía respecto a otras mujeres bolivianas en la Argentina, y en 1986 migra hacia La Paz para "intentar" estudiar y trabajar. En ese momento, Jimena se encontraba en pareja con un compañero de la secundaria que trabajaba en una empresa minera que debió cerrar como efecto de la crisis económica a partir de lo cual decidió migrar a Buenos Aires activando redes familiares extensas 
en el país. Durante los primeros meses de su estadía en la Paz, Jimena descubre que estaba embarazada, una notica que produce la interrupción de su proyecto personal de migración interna y educativa. No ocurre lo mismo con el padre de su hija que continúa su proyecto migratorio "igual yo no quise... digamos, interferir en el plan de él (...) ya tenía todo hecho, o sea, el pasaporte, el pasaje". Decidieron casarse "a las apuradas" y él viajó a Buenos Aires. Un año y medio después de su partida, Jimena recibe el dinero para la compra de un pasaje y una carta donde la invita a instalarse juntos. "Él ya había ahorrado", cuenta Jimena, por medio de su trabajo en el mantenimiento de unas piletas de Ezeiza $^{3}$ donde le ofrecieron construirse una casa. Jimena y su hija se trasladan a Buenos Aires a los pocos días de recibir el dinero y la carta.

En el caso de Viviana, es la última hija de una familia con una larga experiencia migratoria hacia la Argentina. El pueblo donde nació está localizado a dos horas de Posadas ${ }^{4}$. A diferencia de Jimena, todos/as sus hermanos ya habían migrado en una tradición familiar que se remonta a su papá "Siempre hubo algo que me conectaba... digamos, a Argentina". A pesar de vivir en una zona fronteriza, el destino de sus hermanos y su padre siempre fue Buenos Aires para trabajar en el sector de la construcción. En este sentido, la migración a la ciudad porteña era parte de un horizonte de posibilidades cercano y disponible. Viviana termina la escuela secundaria en Paraguay y no tiene experiencia laboral. En esta época sus padres dejan de trabajar por su edad avanzada. La inactividad de sus padres debía ser solventada por las remesas de los hijos e hijas migrantes en Buenos Aires debido a una historia laboral en la informalidad que los excluye de los beneficios de una jubilación. Sus hermanos varones trabajaban (y continúan haciéndolo) en Buenos Aires en la construcción. Sus otras dos hermanas vivían también en Buenos Aires, una realizaba trabajos de costura en su casa, la otra se

\footnotetext{
${ }^{3}$ Partido de la provincia de Buenos Aires a $35 \mathrm{~km}$. de la capital.

${ }^{4}$ Capital de la provincia de Misiones, fronteriza con Paraguay.
} 
dedicaba al cuidado no remunerado de su hogar, después de muchos años de haber sido empleada doméstica. Viviana fue la última en partir, pero su migración fue considerada por toda la familia como la mejor estrategia, ya que permitía reducir costos de mantenimiento del hogar de origen y sumar un ingreso. Migrar, para Viviana, significó iniciar su trayectoria laboral. Emprendió el viaje con 19 años, en el año 2007. Su hermano la recibe en su casa y a partir de los contactos de su familia rápidamente obtiene un empleo como empleada doméstica "cama adentro" en un barrio cerrado del norte de la Provincia de Buenos Aires.

\section{b. La valoración del estudio}

Teniendo en cuenta el objetivo de la investigación, la interpelación a las entrevistadas como enfermeras y el tipo de trayectorias seleccionadas, el estudio formal ocupa un lugar central en los relatos de las entrevistadas.

En primer lugar, es importante recordar que no todas las migrantes en Argentina pueden llegar a ser enfermeras. Existen barreras visibles $e$ invisibles que moldean las aspiraciones y los deseos que se encarnan en diferentes trayectorias laborales. En términos formales, según la normativa nacional, el ejercicio y el estudio de enfermería en cualquiera de sus tres niveles (auxiliar, técnico y licenciado) requiere de un título de estudios secundarios, lo cual implica credenciales académicas que no están distribuidas universalmente entre la población migrante en el país. Según datos del Ministerio de Trabajo (MTEySS) basados en una encuesta realizada en el 2011, la proporción de migrantes que tienen hasta nivel primario incompleto (13,5 por ciento) es más del doble que la de los ciudadanos nativos de la misma edad (OIT, 2015). Dentro de la población extranjera, los perfiles educativos son bastante disímiles: los inmigrantes de países limítrofes cuentan con niveles educativos en promedio inferiores a los de la población total de la Argentina, mientras que la población migrante regional no

${ }^{5}$ Modo de nombrar al empleo sin retiro. 
limítrofe posee perfiles educativos más elevados que los del promedio nacional (Benencia, 2012).

De esta manera, Viviana y Jimena al migrar con diploma de estudios secundarios representan casos excepcionales dentro de la población migrante argentina. Si bien no se trata de proyectos migratorios educativos, la posibilidad (mítica en muchos casos, real para ellas) de poder continuar sus estudios en el nivel terciario es mencionada por ambas como un deseo constante.

En el caso de Jimena, transcurre un período largo de tiempo entre la finalización de sus estudios secundarios y el inicio efectivo en Argentina de la carrera de enfermería. Como ha sido señalado en su relato, es la maternidad lo que le impide la continuación de su proyecto y la orienta a desplazarse hacia Argentina. "Después estoy yo, que estudié acá, terminé el secundario, pero vine acá, me quedé embarazada, no pude seguir estudiando". El valor del estudio formal, de la lectura, se hace presente desde la caracterización de su familia de origen. Jimena describe a sus padres como personas con una participación en la esfera pública "eran muy respetados". Su madre fue creadora de un "club de madres" produciendo tejidos artesanales. Su papá se había formado como ferroviario por correo. Jimena lo recuerda como "muy lector" "tenía cajas de libros". Las construcciones de género organizan los recursos familiares en relación con el estudio. Todos sus hermanos varones estudiaron después de la secundaria: la prioridad del dinero familiar era para la formación masculina. Los hermanos de Jimena se desempeñan como personal de las fuerzas armadas, docentes, ingenieros y arquitectos. Las mujeres han estudiado "por su propia voluntad" como lo expresa Jimena, lo que significa que no han tenido ayuda familiar. Una de sus hermanas es directora de escuela.

Nos criamos en una época diferente, mi mamá y mi papá eran grandes, entonces la prioridad a la hora de orientar los recursos, a quién debería estudiar, estaba orientado a los varones. De hecho, mi mamá me dijo: "Vos si querés estudiar, trabajá. Porque tus hermanos están en la facultad, 
la prioridad son los varones, porque son el sostén de la familia.

A lo largo de la entrevista, Jimena define el largo período en el que no estudió como un alejamiento del destino familiar. Así, menciona en reiteradas ocasiones que sus hermanos la incentivaban a estudiar porque ella "debía" estudiar. A diferencia de otras experiencias migratorias, donde el desplazamiento se realiza para estudiar, en el caso de Jimena el movimiento migratorio es el que trunca el proyecto educativo. Otra situación no prevista lo pospone aún más: al poco tiempo de llegar a la Argentina, el padre de su hija fallece en un accidente. Jimena, sin redes propias (las de su familia política se interrumpen con el fallecimiento) debe solventarse económicamente $y$ en soledad teniendo como único recurso su propio salario. Es en este momento que decide ingresar a trabajar como empleada doméstica en casas particulares, un trabajo de fácil acceso y de remuneración inmediata (a diferencia de otros proyectos de micro emprendimientos en los que había pensado). Si bien fue una opción posible, Jimena descarta la posibilidad de la vuelta a Bolivia tanto por el peso simbólico del "fracaso" del proyecto migratorio como por las escasas posibilidades que percibe en Bolivia "Y entonces yo pensé, somos tantos hermanos, ¿qué voy hacer en Bolivia?, me quedo acá, por lo menos mi hija si... va a cobrar algo... va a poder estudiar. Eso pensé". Si bien no envía remesas, la totalidad de su salario lo destina al alquiler de una vivienda y a la contratación de una cuidadora para su hija.

En el caso de Viviana, la continuación de los estudios es mencionada como una de las motivaciones para emigrar hacia la Argentina junto con la necesidad de un empleo remunerado. Viviana cree que en Buenos Aires se multiplican las posibilidades de realizar algún curso terciario. En su relato, el hecho de haber terminado la escuela secundaria se convierte en un elemento central de la definición de sí misma, especialmente en la comparación con otras mujeres migrantes. Diferentes escenas de su biografía están atravesadas por esta imagen. Por ejemplo, 
cuando comienza a trabajar como empleada doméstica sin retiro Viviana destaca que su empleadora se identificaba con ella por sus ganas de seguir estudiando (trabajaba de lunes a viernes en un barrio lejano de su lugar de residencia, lo cual hacía muy difícil poder realizar cualquier tipo de estudio). Cuando Viviana, por decisión propia, circula hacia un empleo con retiro, inicia una serie de cursos de formación profesional ofrecidos por diferentes instituciones privadas tales como un curso de operadora de PC y otro de liquidación de sueldos y jornales. A los pocos años de estar en la Argentina, forma una pareja con quien tiene una hija. La última etapa del embarazo y el primer año de su hija, Viviana deja de trabajar, en gran parte por sugerencia de su marido, temática que profundizaremos en el siguiente apartado.

La formación continua y sus deseos de seguir estudiando le permiten a Viviana posicionarse en un espacio diferente al de otras mujeres con las que comparte el tipo de empleo pero que define como más "vulnerables". Según su percepción, la menor educación formal modifica, entre otras dimensiones, el trato que reciben de sus empleadoras.

Porque a mí me adoraba, me respetaba, me hablaba bien. Pero yo veía que... también había una chica que era paraguaya, que a diferencia conmigo no había estudiado, era... muy chiquita, creo que tenía 20 años y... era muy vulnerable y... Y nada, estuve ahí trabajando... a mí me querían, me adoraban, era la mejor (Risa leve) Pero porque se daba cuenta que no podía... yo no me iba a dejar...

Sin embargo, la importancia del estudio para Viviana repercute al mismo tiempo en una visión deteriorada de sí misma que no se revierte hasta que no inicia la carrera de enfermería. En su relato, Viviana decide continuar con sus estudios terciarios luego de una breve separación de su marido. En ese momento, no trabajaba a causa de su reciente maternidad y la separación significó una reflexión sobre su situación actual. En sus propias palabras "no entendía cómo terminé asi". La metáfora de "seguir 
adelante", los deseos de progresar y de "ser alguien en la vida" por ella y por su hija tienen un vehículo: la obtención de un diploma terciario. Estudiar significa también ganar autonomía.

yo quería salir adelante por... cuenta propia, ser alguien en la vida, tener una profesión, tener un título y... poder darle a mi hija un futuro mejor" ... por mi cuenta y si... si era... O sea, si es posible con mi marido al lado, pero si no era posible eso, con que... conmigo misma, de valerme por mí misma y para darle un futuro mejor a ella.

Como es posible apreciar, en los dos casos analizados existe una relación con los estudios que explica, en parte, el itinerario de sus trayectorias. Tanto en las características familiares de origen (valoración y acceso a la educación formal media y terciaria), en sus propias credenciales educativas al momento de migrar y en la búsqueda constante de la formación terciaria como una forma de cumplir con un destino prefijado, como una forma de reorientar hacia cierta "normalidad" sus propias trayectorias de vida.

c. Redes, apoyos y gestión de los cuidados

Las redes sociales pueden ser consideradas un elemento clave de la teoría migratoria contemporánea en tanto sitúa a los y las migrantes en un entramado de relaciones sociales que organiza el desplazamiento migratorio y las estrategias futuras (Herrera Lima, 2000; Pedone, 2010). En este artículo, sin embargo, no me interesa analizar las redes sociales para el traslado migratorio sino la importancia de su presencia (o ausencia) como un elemento clave en el análisis de las trayectorias de las entrevistadas. En el caso de las trayectorias laborales y educativas de las mujeres, sin importar su condición migratoria, las redes son fundamentales para ampliar las formas posibles de gestión del cuidado de sus familias de tal modo de poder habilitar, material y simbólicamente, la ausencia del hogar de las mujeres y su presencia en actividades laborales y educativas. Desde esta perspectiva, los casos de Jimena y Viviana son divergentes, pero ambas nos muestran la imbricación 
necesaria entre los géneros, los cuidados y las temporalidades (Bessin y Gaudart, 2009).

Como se ha indicado, Jimena se desplaza a Buenos Aires en un clásico movimiento de reunificación familiar, con el fin de poder vivir con el padre de su hija. Sin embargo, el hecho trágico de su muerte en un accidente de tráfico reconfigura el escenario local y Jimena se queda sin el manto de protección que significan las redes sociales por el rechazo de la familia de su pareja y la ausencia de sus propias redes familiares en el país. Alquila una casilla, y desde ese momento sus decisiones se orientan a poder mantener la reproducción de su hogar a partir de su ingreso monetario. Jimena trabaja esos años "limpiando casas de familia". A su hija la dejaba en una guardería comunitaria. Su sueldo alcanzaba justo para cubrir gastos básicos y los cuidados remunerados de su hija. No tenía posibilidades de ahorrar. Sus primeros trabajos fueron cerca de su casa, en Ezeiza, para poder volver a tiempo para cuidar a su hija. Cuando creció un poco más, pudo insertarse en empleos domésticos en la Ciudad de Buenos Aires donde obtuvo mayores ingresos lo cual, sumado al acceso de su hija a las instituciones públicas de nivel preescolar y primario, le permitió poder ahorrar algo de dinero. Logró, en algunos años, poder comprar un terreno y construir su casa. A los 27 años, Jimena se volvió a casar. En sus palabras "no enamorada, eso fue... buscando más bien compañía". El matrimonio, además de compañía, ensanchó su horizonte de posibilidades al habilitarle nuevas estrategias vedadas en su anterior situación. Jimena enfatiza en la entrevista que, si no era el amor lo que la acercó a su actual marido, tampoco era la conveniencia en términos económicos. Nunca dejó de trabajar, su empleo es la base de su autonomía. Tuvo una segunda hija de este segundo matrimonio, pero esta vez contaba con la ayuda no rentada de la abuela de su hija y de su marido (que trabajaba por la mañana), por lo que pudo continuar con sus trabajos. Es en este contexto en el que Jimena decide continuar sus estudios. Por primera vez desde su llegada a la Argentina cuenta con personas cercanas que le permiten ausentarse de su hogar una mayor 
cantidad de horas de las que trabaja (las cuales también reduce ante la posibilidad de contar con otro ingreso en el hogar).

De esta manera, el relato de Jimena brinda algunas pistas para analizar la influencia de la gestión de los cuidados domésticos y la presencia de redes sociales como dimensiones centrales en las decisiones laborales y vitales. Jimena inicia su experiencia como trabajadora migrante en la Argentina con una hija pequeña y sin los recursos que suelen circular en las redes de migrantes (informativos, afectivos, de cuidado, materiales). Sus opciones laborales están condicionadas por esta situación: necesita trabajo urgente y el empleo doméstico permite esta inmediatez. La primera inflexión en sus posibilidades laborales es consecuencia de la oferta educativa pública de nivel preescolar. Para Jimena, significa un gasto menos y mayor cantidad de horas disponibles que invierte en viajar a la Ciudad de Buenos Aires donde los salarios para las empleadas domésticas son significativamente mayores. La segunda inflexión es su matrimonio que suma un salario al hogar y que le permite acceder a redes de cuidado familiares de las que no había gozado a lo largo de su permanencia en Buenos Aires. La disponibilidad económica para poder disminuir su carga laboral, y la temporal que resulta de poder contar con una red familiar de cuidados son las condiciones necesarias para que Jimena decida iniciar su formación como enfermera.

El caso de Viviana es diferente. Migra en un contexto de redes migratorias y familiares, lo que también puede explicar los tiempos más cortos, si se los compara con lo que ocurre con Jimena, entre la migración y el inicio de su carrera de enfermera. Cuando llega al país, se queda viviendo un tiempo en la casa de su hermano, pero rápidamente comienza a trabajar como empleada doméstica sin retiro a través de la recomendación de sus hermanas. Los fines de semana se instala en la casa del mismo hermano que la recibió y luego alquila una con otra hermana durante un tiempo hasta que ambas se ponen en pareja y se autonomizan. Viviana empieza a convivir con su actual marido. En ese momento, como se detallará en el apartado siguiente, 
Viviana intenta salir del empleo doméstico a partir del acompañamiento, afectivo y monetario, de su pareja. Sin embargo, en ese camino Viviana también percibe una pérdida de su autonomía. En el 2009 Viviana queda embarazada lo que la lleva a abandonar sus empleos por sugerencia de su marido. La informalidad de las relaciones laborales no le permiten solicitar una licencia. Al respecto, Viviana señala que:

\begin{abstract}
en realidad no sabía bien cómo hacer, pero no quería quedarme mucho tiempo en mi casa. Pero por otro lado también... quería disfrutar de ese momento con mi hija. Y... es más, llegué a un punto en que he ido a trabajar un par de días y demás. Después decidí que no. Dice mi marido: "Quédate acá, vos que podés y disfrutala", porque él trabajaba...
\end{abstract}

El salario de su pareja le brinda la posibilidad de no trabajar de manera remunerada lo que, sumado a su reciente maternidad, la llevan a decidir una salida del mercado de trabajo. Después de una breve separación, decide volver a ocuparse en un empleo remunerado en miras de adquirir una mayor autonomía e iniciar el estudio de enfermería. La presencia del padre de su hija, y su involucramiento en el cuidado de su hija, le permite trabajar algunas horas como empleada doméstica mientras estudia enfermería profesional.

Como es posible apreciar, en ambas historias la maternidad es un evento central que reorganiza el resto de las esferas de actividad de las entrevistadas, lo que no sucede con los padres que continúan con sus proyectos migratorios y/o laborales. La maternidad de Jimena la lleva a migrar. Viviana decide dejar de trabajar por sugerencia de su marido. Convertirse en madres condiciona las trayectorias y siempre supone la realización de ciertas actividades y la disponibilidad mental y material para solucionar las nuevas demandas. También, otorga una nueva legitimidad a cierto "sacrificio" maternal expresado en extensas jornadas laborales a las que luego se sumarán las horas de estudio. En este sentido, las redes sociales de cuidado disponibles 
son un recurso indispensable para alivianar la responsabilidad moral que en nuestras sociedades se derivan de la maternidad, ante la dificultad de acceder a recursos estatales y privados enfocados al cuidado de niños y niñas. Asimismo, generan un tiempo "extra" por fuera de la temporalidad de la responsabilidad que es la condición necesaria para poder iniciar el estudio de enfermería. Es a partir de cierta redistribución del trabajo de cuidado no remunerado en el hogar, históricamente desigual en detrimento de las mujeres, que Jimena y Viviana cuentan con la disponibilidad material y simbólica para estudiar enfermería. Sin embargo, para las dos la inserción en redes también es percibida como una amenaza a una autonomía generada y reproducida a partir de la actividad laboral. Quizás se deba a que, en los casos analizados, el ensanchamiento del tiempo disponible se genera en gran parte a partir de las parejas de las entrevistadas. De esta manera, la presencia activa de los varones y redes familiares en el cuidado doméstico no remunerado resulta un elemento central que permite la proyección de nuevos horizontes en las trayectorias analizadas.

\section{d. El rol de trabajo doméstico}

El hecho de que las protagonistas se desempeñaron como trabajadoras domésticas, permite definir las carreras laborales como una "circulación entre empleos de cuidado". En este apartado serán analizados los significados en torno a esta tarea y su relación con el ejercicio de la enfermería.

Como ha sido señalado en otros trabajos (Mallimaci $y$ Magliano, 2016) el trabajo doméstico significa en las trayectorias de mujeres migrantes en Argentina la posibilidad de un ingreso rápido al mundo del trabajo. Según los datos estadísticos, el trabajo doméstico es un nicho de mercado privilegiado para las mujeres migrantes, especialmente las paraguayas y peruanas. Entre las primeras, un 69 por ciento están ocupadas en este tipo de empleo. Si miramos el total de las extranjeras empleadas en el empleo doméstico, más de la mitad provienen de Paraguay (52 
por ciento). Las bolivianas representan al 8,9 por ciento. De esta manera, si bien se trata de una ocupación relevante para el empleo femenino en general (17 por ciento del total de asalariadas de todo el país) entre las mujeres migrantes, especialmente paraguayas y peruanas, representa el nicho sectorial en el que se insertan mayoritariamente (Maguid, 2011). Las cifras estadísticas indican que, en el Área metropolitana de Buenos Aires (AMBA), el $42,6 \%$ de las migrantes internacionales del AMBA se insertan en el trabajo doméstico (Rosas et alii, 2015).

Para muchas mujeres migrantes, sin importar las trayectorias laborales previas, el ingreso al mundo del trabajo se realiza vía el empleo doméstico. En esta línea, Tizziani (2011) analiza el empleo doméstico en Argentina y discute una visión expandida sobre el trabajo en casas particulares que lo percibe como una entrada siempre provisoria al mundo laboral hasta alcanzar "el trabajo deseado". En su investigación Tizziani muestra como las empleadas domésticas se instalan por largos períodos de tiempo en sus empleos lo que les posibilita circular horizontalmente por diferentes posiciones, pero mejorando las condiciones de trabajo y los salarios. Esta permanencia en el trabajo doméstico no depende de las credenciales educativas ni de las experiencias laborales previas que posean las mujeres (Maguid y Bruno, 2010).

Las mujeres cuyos relatos son analizados en este trabajo, pertenecen al grupo menos frecuente de mujeres que ingresan al mercado de trabajo por la vía del trabajo doméstico, y que a partir de la obtención de un título profesional circulan hacia empleos vinculados con la profesión. Sin embargo, en sus relatos la enfermería y el trabajo doméstico no son percibidos como dicotomías entre un trabajo "bueno y deseable" y otro "malo y no deseable". Por el contrario, la relación entre ambos se configura en una línea causal y acumulativa en la cual el trabajo doméstico es la plataforma desde la cual es posible multiplicar las opciones laborales y vitales. Esto se realiza en dos sentidos principales. El primero, al permitir la obtención de recursos económicos para poder iniciar y continuar la formación (pago de aranceles, de uniformes y elementos técnicos). El segundo, al considerar el 
empleo doméstico como el ámbito en el cual se empieza a acumular experiencias de cuidado que les permiten definir a la enfermería como una opción alcanzable. De esta manera, el empleo doméstico adquiere una importancia vital en las trayectorias.

En un artículo anterior, hemos señalado con María José Magliano la existencia de dos formas típicas en las que el empleo doméstico surge como una opción en las trayectorias de las mujeres migrantes: como opción laboral emergente en el contexto de llegada, asociado a la migración boliviana, y el empleo doméstico como orientador del proceso migratorio más relacionado con peruanas y paraguayas (Mallimaci y Magliano, 2016). Las historias de Jimena y Viviana expresan estas tradiciones más amplias. La elección de Jimena por el trabajo doméstico es estratégica y surge como respuesta a la muerte del padre de su hija. Por el contrario, Viviana migra con el proyecto del empleo doméstico como orientador de su migración.

A lo largo de su permanencia en el país, ambas rotan entre diferentes empleos domésticos mejorando sus condiciones laborales. De esta manera, Viviana pasa de empleos domésticos sin retiro a empleos por horas que abandona sólo cuando comienza a trabajar como enfermera. Jimena modifica la localización de sus trabajos y la extensión horaria mejorando su salario y, al igual que Viviana, cambia el empleo doméstico por el empleo como enfermera. De esta manera, se presencia una movilidad horizontal dentro del trabajo doméstico que antecede a la movilidad vertical producida en el acceso a la enfermería. Viviana describe muy bien la movilidad horizontal al describir las diferencias entre un trabajo sin retiro a uno con retiro: el primero permite generar ahorros con mayor celeridad, pero

...es como una prisión, uno no puede tener vida propia, no puede tener privacidad, no puede llorar tranquila... Es estar a la orden de alguien siempre. Si bien me trataron muy bien en todos mis trabajos, eh... nada, eso. 
En términos de representaciones sociales hegemónicas, el empleo doméstico es un empleo poco valorado socialmente, acercándose a lo que puede considerarse un "trabajo sucio" (Arango Gaviria, 2011). Sin embargo, tanto Jimena como Viviana optan por construir otros significados sobre estos empleos que, al momento de realizar las entrevistas, formaban parte de su pasado. La relación con el empleo doméstico se define de un modo pragmático por fuera de toda valoración moral. Es decir, para las entrevistadas el empleo doméstico es aquello a lo que podían acceder en un tiempo corto acorde a sus condiciones de posibilidad y su posición en la sociedad. La importancia que tiene para ellas el estudio refuerza la inevitabilidad del empleo doméstico: sin estudios son estos los empleos que les tocan.

Por otra parte, la visión que tienen sobre su experiencia en el trabajo doméstico se sostiene en la comparación con las condiciones de trabajo de otros empleos que fueron "posibles para ellas". En el caso de Viviana intenta buscar otros tipos de empleo cuando comienza a convivir con su pareja, trabaja como operaria de una fábrica y luego como vendedora de productos de belleza por catálogo. Sin embargo, ambos empleos presentan condiciones laborales que son percibidas por Viviana como más precarias que el trabajo en casas particulares. Por ello, después de estos intentos y el período de inactividad ligado a la maternidad, Viviana vuelve a insertarse como trabajadora doméstica. Lo mismo sucede al comparar sus trabajos como empleadas domésticas, con años de permanencia, con las primeras ofertas de trabajos precarios como estudiantes de enfermería. Ambas, al iniciar la carrera de enfermería, tuvieron posibilidades de ser contratadas en instituciones de cuidado o en empresas que prestan servicios de cuidado domiciliario. Las dos rechazaron estas ofertas debido a que el salario ofrecido era menor del que estaban percibiendo en sus empleos y las jornadas laborales más extensas y rígidas. La flexibilidad del trabajo en casas particulares bajo la contratación "por horas" se revaloriza por su flexibilidad que les permite manejar las jornadas laborales en relación con el tiempo de estudio, los exámenes y las prácticas que deben realizar y el 
constante trabajo de cuidado no remunerado que deben realizar en sus hogares.

Sin duda alguna, las condiciones en las que ejercían su trabajo como empleadas en casas particulares vulneraban una serie de derechos laborales básicos al tratarse de relaciones laborales no registradas. Sin embargo, se trata de una situación que no es exclusiva de este tipo de empleos, sino que se repite en la totalidad de los empleos que tuvieron disponibles.

La resignificación del empleo doméstico como "opción" laboral no supone la negación de las relaciones asimétricas y la precariedad de la relación laboral. Tampoco significa que las entrevistadas no mencionen aspectos negativos del empleo doméstico. En este sentido, se subraya el tiempo invertido en los viajes hacia sus trabajos (vinculada a la distancia que suele haber entre la localización de los hogares que demandan empleos domésticos y la de los hogares de las empleadas). El hecho de que se trata de empleos realizados dentro del espacio doméstico es otro de los aspectos mencionados como negativos, al generar la posibilidad de un límite poco claro entre el empleo y las relaciones personales o afectivas (Gorban, 2012). Sin embargo, los aspectos positivos se imponen a la hora de decidir continuar sus trabajos como empleadas domésticas a lo largo del tránsito por las carreras de enfermería.

Un elemento central para comprender la significación del empleo doméstico por parte de las entrevistadas es su vinculación simbólica con la enfermería, especialmente al nivel de las prácticas que suponen ambos trabajos. Ambas entrevistadas realizan en sus relatos esta conexión simbólica entre algunas de las actividades que realizaban como trabajadoras domésticas y su actual profesión como enfermeras. Jimena destaca la dimensión relacional y afectiva que supone el trabajo doméstico, ya sea de limpieza o de cuidado en sentido estricto del término y su relación con el lazo que debe establecer la enfermera con sus pacientes.

Las grandes diferencias... Por ahí... eh... empleada doméstica a lo mejor, de alguna manera era... colaborás o 
ayudás, acompañás... porque también acompañás a la otra persona, porque no es que sos un instrumento que va y limpia. Esto de poder..., somos personas y hablar $y$ compartir. Por lo menos con las señoras que yo trabajé... me confiaban cosas, éramos más compañeras. De hecho, a mí me dolió irme y a ellas también... no querían que me fuera, porque sabían que yo me estaba yendo para mejorar en lo personal...

Viviana encuentra en algunos de sus trabajos como empleada doméstica (en los que las tareas de cuidado y las actividades de limpieza tienen un límite difuso) el germen de una predisposición al cuidado que define como propio de la enfermería:

Y ahí... estuve... casi dos años más o menos... hasta que... fue también como una conexión, ahora lo recuerdo... con mi profesión ahora, porque había una chica, o sea, la hija de mi jefa, porque era separada, que era cuadripléjica... Perdón, era parapléjica... porque no movía la parte de las piernas y demás. Tuvo que aprender todo... y ella no tenía movilidad fina. Yo la asistía, y a ella... en un momento me... mucha vergüenza... Era muy independiente la chica, pero me pidió que le ayude a ponerse una sonda vesical... y... No me daba impresión ni nada...Y me interesaba el cuidado. O sea, siempre... el cuidado hacia el más vulnerable, digamos.

De esta manera, los relatos incorporan al empleo doméstico como un elemento necesario de sus trayectorias al estructurar el presente laboral. Empleo doméstico y enfermería son parte de una misma circulación laboral. Si bien el estudio y ejercicio de la profesión de enfermería es definido como "una mejora personal" al tratarse del acceso a una profesión, esto no significa reconocer características supuestamente aversivas (o sucias) en las actividades que involucran el trabajo doméstico. Sin duda, como lo veremos en el apartado que sigue, esta operación valorativa se 
relaciona con que las actividades propias de la enfermería suponen prácticas de higiene y cuidados profesionales.

e. La elección por la enfermería

En las dos historias analizadas, el inicio de la carrera de enfermería realiza la predisposición al estudio que las entrevistadas señalaron como un rasgo personal y esencial de sus biografías. En este sentido, ante la pregunta sobre los motivos de la elección de la carrera, Viviana remite a la necesidad de completar su trayectoria educativa dada su condición de "buena alumna".

E: ¿Cómo se te ocurrió estudiar enfermería?

Viviana: Eh, bueno, fue así... Tengo una amiga, que terminamos el colegio juntas, y que... las dos fuimos -creo que ahora no lo utilizan- pero terminamos alumnas distinguidas del curso, fuimos las mejores alumnas de nuestro curso. Y había chicos que no... que uno... la verdad que no daba dos pesos por ellos. Sin embargo, nos enterábamos de que... tenían una profesión, estudiaron... Si bien es en cosas privadas que por ahí uno... ellos pagan y es más fácil y todo. Y nos decíamos: "No puede ser que nosotras terminamos asi”"

En segundo lugar, la elección de enfermería entre otras carreras estuvo basada en diversas representaciones sociales sobre la profesión. En este sentido, ambas hacen referencia a ciertas creencias compartidas que tenían sobre la carrera que permitieron definirla como una carrera "chica" y "fácil" lo que la convertía en una meta posible de ser alcanzada.

Viviana: Enfermería, por ahí sí lo veía como algo más chiquito. Porque uno habla mucho de prejuicios y el prejuicio está en uno muchas veces. Y lo veía como algo más acotado, digamos. (...) Hay una idea de que es muy fácil, de que cualquiera puede hacerlo, de que no se necesita de muchos conocimientos. Yo creo que eso 
también viene de... de cómo se formó enfermería anteriormente.

Jimena: Después dije bueno, voy a estudiar... yo pensé lo más fácil y lo más rápido es enfermería. Porque uno tiene prejuicios. Si yo a vos te pregunto qué pensás de enfermería, ¿qué me dirías?... ¿La enfermera qué hace? Toma la presión y pone inyecciones. Entonces me metí por eso.

De esta manera, representaciones comunes sobre los contenidos de la profesión como un saber menor dentro del campo médico orientan las decisiones de Viviana y Jimena. Es importante señalar que ambas destacan como parte fundamental de su formación la puesta en discusión de estos prejuicios iniciales para construir una nueva consideración sobre la enfermería como un saber específico y autónomo vinculado al cuidado profesional de los/as pacientes.

Un tercer elemento que se visibiliza como propulsor de la elección de la carrera se relaciona con aspectos vinculados a la dimensión vocacional de la profesión. Como he analizado en otros trabajos (Mallimaci, 2016, 2017), en la investigación mayor las estudiantes y profesionales de enfermería entrevistadas suelen construir definiciones vocacionales sobre la profesión. En este sentido, se señala de modo recurrente la existencia de una predisposición esencial hacia el trabajo de enfermería traducido en una forma de vinculación con las personas dependientes y cierta inclinación al cuidado. Jimena y Viviana, repitiendo modelos que se repiten en otras entrevistas, señalan el ámbito familiar como el espacio de aprendizaje donde esta vocación suele ser evidenciada. La figura de la madre emerge como emblema de una experiencia de cuidado vocacional, que "no pide nada a cambio". El cuidado se comprende como una forma de relacionarse con los/as otros/as.

Jimena: Este... y además aprendí de mi mamá a cuidar al otro, porque mi mamá era una mujer muy solidaria. Teniendo tantos hijos y todo... ella era... siempre había un 
plato más para el turista que pasaba..., tenía un techo, tenía un plato de comida.

Viviana: Mi mamá era ama de casa y... también hacía trabajos de costura. En mi casa, sí. Y... era... una especie de enfermera... como las de antes, aplicaba inyecciones y demás. Que había aprendido de... de un doctor... conocido, digamos. Entonces hacía prestaciones... eh... hacía a familiares y si alguien necesitaba lo hacía, sin pedir nada. Ya... creo que lo mío viene por ese lado, digamos.

Me interesa señalar particularmente que, en el caso de las dos historias analizadas en este artículo, esta predisposición al "cuidado como vocación" y "la preocupación por los otros" que supone el trabajo enfermero, se inicia en los trabajos anteriores ejercidos como empleadas domésticas. Tal como se ha señalado en el apartado anterior, existe en las entrevistadas la percepción de que han realizado una carrera en el trabajo de cuidados vinculando saberes y prácticas del empleo doméstico con aptitudes que consideran necesarias para la enfermería: la predisposición a la escucha y al cuidado; el saber hacer la higiene corporal y la limpieza se vivencian como prácticas aprendidas que pueden ser competencias en empleos remunerados. El cuidado como vocación, cuyo modelo es el trabajo en el hogar, se traslada al ámbito doméstico de otros/as y se recupera como destreza laboral: es posible cuidar de espacios ajenos y personas no familiares.

De esta manera, las entrevistadas optan en sus relatos por ligar las actividades profesionales de la enfermería con saberes y competencias aprendidas en el desempeño de sus funciones como empleadas domésticas. De modo similar a lo señalado por Tizziani (2017) para las y los trabajadores de limpieza, la acumulación de experiencias en torno a las actividades de cuidado les permite reivindicar la adquisición de una serie de saberes y competencias (técnicas y relacionales) que las vuelven idóneas para el estudio de la enfermería. De este modo, existe una conexión simbólica entre las tareas de enfermería y las tareas efectivamente realizadas como 
trabajadoras domésticas remuneradas. La experiencia no profesional de cuidado en el trabajo doméstico (sobre los cuerpos, las personas y sobre los espacios) se vivencian como modelos cercanos a las actividades de cuidados profesionales.

Creo que es posible interpretar en estas operaciones discursivas una apuesta valorativa con el fin de ganar cierta respetabilidad (Skegg, 2015) que las lleva a naturalizar los saberes y prácticas relacionados con el cuidado al construir genealogías de cuidado familiares en tanto dones femeninos que se heredan de generación en generación. Pero a la vez, se trata de una reivindicación sobre la posibilidad de acumular experiencias y ampliar las competencias y profesionalizarlas a lo largo de sus carreras laborales.

\section{Conclusiones}

El análisis realizado muestra como las trayectorias de las entrevistadas circulan entre diferentes actividades de cuidado constituyendo la realización de una "carrera informal" en el sector. Ser enfermeras supone una dinámica ascendente en términos de consideración social y condiciones de trabajo, tanto por las calificaciones que requiere la actividad, el prestigio social de su ejercicio en relación con el del trabajo doméstico, las condiciones laborales y seguridades asociadas al empleo formal, la institucionalización de las relaciones laborales y el tipo de espacio (no doméstico) en el que se realiza. Sin embargo, desde la perspectiva de las entrevistadas, entre el trabajo doméstico y la enfermería no existen experiencias dicotómicas, sino que ambos empleos son percibidos formando una misma cadena causal y acumulativa. Convertirse en enfermeras, en esta narrativa, supone haber sido empleadas domésticas: la elección de la profesión está marcada por la experiencia no profesional en el trabajo doméstico, donde se adquieren habilidades de cuidado en contextos no familiares (que es marcado como el origen principal del "saber cuidar"). Además, es posible interpretar la revalorización del empleo doméstico como una manera de revalorizarse a sí mismas 
y dar cuenta de una profunda reflexividad sobre las posibilidades del mercado de trabajo argentino.

Existen algunas condiciones que aparecen como necesarias para poder circular entre estas diferentes actividades que los relatos recogidos en este trabajo colaboran a visibilizar. Primero, la importancia para las protagonistas del valor del estudio, que se expresa tanto en las credenciales educativas que poseen al migrar como en la convicción de que la vía para cumplir sus deseos de ascenso social es la posesión de un título terciario o universitario. Segundo, la presencia de redes sociales y familiares que les permiten gestionar sus tiempos productivos y reproductivos de manera tal de generar el plus (temporal y material) necesario para poder estudiar una carrera. En tercer lugar, debe señalarse que la carga económica de estas mujeres migrantes es menor a algunas de sus compatriotas al no tener que sostener una familia transnacional ni tener que remesar en sus hogares de origen. Tampoco sostienen económicamente otras familias en Argentina, por lo tanto, en ambos casos, los proyectos tienen como único espacio destinatario el lugar en el que viven en la actualidad y sus familias nucleares, lo cual amplía sus posibilidades.

En términos de transformaciones en las relaciones de género, las mujeres cuya trayectoria relatamos en este artículo han valorado su autonomía económica y de proyectos vitales (condensadas en la valoración del estudio) a lo largo de sus vidas, lo que las ha llevado a tomar decisiones fundamentales. Sin duda alguna, existe una enorme diferencia en relación con sus madres que representan modelos más tradicionales de mujeres y domesticidad. Para Jimena y Viviana, la maternidad no es suficiente para sentirse realizadas, siendo la profesión de enfermeras un elemento central de sus vidas actuales. Sin embargo, no podría decirse que se trata de transformaciones que son fruto de la migración, sino que se concretan y proyectan gracias a los desplazamientos migratorios. Aún así, el modelo de cuidados que expresan sigue anclado en la naturalización de las mujeres como sujetas aptas para el cuidado. A pesar de la reivindicación de la profesionalización del cuidado, la genealogía 
del "saber cuidar" tiene como origen necesario sus madres y sus hogares de la infancia.

\section{Referencias bibliográficas}

CeRRUTTI, Marcela; BRUNO, Sebastián. La inserción de los migrantes paraguayos y peruanos en el mercado de trabajo del Área Metropolitana de Buenos Aires. Estudios Migratorios Latinoamericanos (60), Buenos Aires, Centro de Estudios Migratorios Latinoamericanos (CEMLA), 2006, pp.265-290.

Benencia, Roberto. Perfil migratorio de la Argentina 2012. Buenos Aires, Organización Internacional para las Migraciones (OIM), 2012.

BESSIN, Marc y GAUDART, Corinne. Les temps sexués de l'activité : la temporalité au principe du genre. Temporalités 9, Paris, 2009 [http://temporalites.revues.org/979 - acceso el 2 feb. 2017].

BORGEAUD GARCIANDIA, Natasha. En las grietas de la dominación. Buenos Aires, Editorial Teseo, 2014.

GORBAN, Débora. Empleadas y empleadoras, tensiones de una relación atravesada por la ambigüedad. Revista Española de Investigaciones Sociológicas $n^{\circ}$ 140, Madrid, 2012, pp.29-48.

Groisman, Fernando y SCONFIENZA, Ma. Eugenia. El servicio doméstico en Argentina. Particularidades y desafíos de un sector relegado (20042012). Carta Económica Regional (25), 2013, pp.151-172.

HERRERA LiMA, Fernando. Las migraciones y la sociología del trabajo en América latina. En: De la Garza, Enrique, Tratado de Sociología del Trabajo, México: FCE, 2000, pp.566-586.

HinOJOSA GoRDONAVA, Alfonso. Buscando la vida. Familias bolivianas transnacionales en España. La Paz, CLACSO, 2009.

MAGUID, Alicia. Migrantes sudamericanos y mercado de trabajo. En: La inmigración laboral de sudamericanos en Argentina. Buenos Aires, OIT, Ministerio de Trabajo, Empleo y Seguridad Social, 2011, pp.109-130.

, Alicia; BRUNO, Sebastián. Migración, mercado de trabajo y movilidad ocupacional: el caso de los bolivianos y paraguayos en el 
Área Metropolitana de Buenos Aires. Población de Buenos Aires vol. 7, n²12, 2010, pp.7-28.

MallimaCi BARRAL, Ana Inés. Migraciones y géneros. Formas de Narrar los movimientos por parte de migrantes bolivianos/as en Argentina. Revista Estudos Feministas 19(3), Florianópolis, setembrodezembro/2011, pp.751-775.

, Ana Inés. Moviéndose por Argentina: Sobre la presencia de bolivianos en Ushuaia. Migraciones Internacionales 6 (23), México, 2012, pp.173-207.

, Ana Inés. Migraciones y cuidados. La enfermería como opción laboral de mujeres migrantes en la ciudad de Buenos Aires. Universitas Humanística, [S.1.], vol. 82, $\mathrm{n}^{\circ}$ 82, Colombia, 2016 [http://revistas.javeriana.edu.co/index.php/univhumanistica/article/vie w/13068 - acceso el 05 ago. 2016].

Ana Inés. Mujeres migrantes y la gestión de los cuidados. La enfermería en el horizonte laboral. En: Borgeaud-Garciandía, Natacha (ed.). El trabajo de cuidado, Buenos Aires (en prensa).

, Ana Inés; MAGLIANO, María José. Migraciones, género y cuidados en Argentina: jerarquizaciones, desigualdades $y$ movilidades. En: MAGLIANO, María José et alii. Los nudos ciegos de la desigualdad. Diálogos entre migraciones y cuidado. Buenos Aires, CONICET, 2016, pp.83-109.

ORGANIZACIÓN INTERNACIONAL DEL TRABAJO. Migraciones laborales en Argentina. Protección social, informalidad y heterogeneidades sectoriales. Buenos Aires, Oficina del País de la OIT para la Argentina, 2015.

PEDONE, Claudia. Cadenas y redes migratorias: propuesta metodológica para el análisis diacrónico-temporal de los procesos migratorios. Empiria Revista de Metodología de Ciencias Sociales n 19, 2010, pp.101-132.

SkEGGS, Beverley. Des femmes respectables. Classe et genre en milieu populaire. Marseille, Éditions Agone, 2015.

TIZZIANI, Annia. De la movilidad ocupacional a las condiciones de trabajo. Algunas reflexiones en torno a diferentes carreras laborales 
dentro del servicio doméstico en la ciudad de Buenos Aires. Trabajo y Sociedad n 17, 2013, pp.309-328.

, Annia. Las ocupaciones de limpieza en el Área Metropolitana de Buenos Aires: territorios y significados en torno del "trabajo femenino" y el "trabajo masculino". En el Seminário Internacional Fazendo Gênero 11 \& 13th Women's Worlds Congress, Florianópolis, Brasil, 30 de julio a 04 de agosto 2017. 